\title{
SZASZ ANALYTIC FUNCTIONS AND NONCOMPACT KÄHLER TORIC MANIFOLDS
}

\author{
RENJIE FENG
}

\begin{abstract}
We show that the classical Szasz analytic function $S_{N}(f)(x)$ is related to the Bergman kernel for the Bargmann-Fock space. Then we generalize this relation to any noncompact toric Kähler manifold, defining the generalized Szasz analytic function $S_{h^{N}}(f)(x)$. Then we will prove the complete asymptotic expansion of $S_{h^{N}}(f)(x)$ and its scaling limit property. As examples, we will compute the generalized Szasz analytic function for the unit ball with the Bergman metric and complex 1,2 and 3-dimensional Kepler manifolds with incomplete Kähler metrics. In addition, three examples about noncompact complete Kähler toric manifolds are discussed, among which are the total space $\mathcal{O}(-1) \rightarrow \mathbb{C P}^{1}$, toric Sasaki-manifolds and Reinhardt Domains.
\end{abstract}

\section{INTRODUCTION}

In [Z1], S. Zelditch relates the classical Bernstein polynomials Be] to the Bergman kernel for the Fubini-Study metric on $\mathbb{C P}^{1}$, and then generalizes this relation to any compact Kähler toric manifold. In this article, we will generalize his idea to any noncompact Kähler toric manifold. The model in the noncompact case is the Bargmann-Fock space, in which the Bernstein polynomials are replaced by the Szasz analytic functions $[\mathbf{S}]$ :

$$
S_{N}(f)(x)=e^{-N x} \sum_{\alpha=0}^{\infty} \frac{(N x)^{\alpha}}{\alpha !} f\left(\frac{\alpha}{N}\right), N>0
$$

Szasz analytic functions are connected to the Poisson distribution in the same sense that Bernstein polynomials are connected to the binomial distribution. In [S], O. Szasz proved that if $f(x)$ is a continuous function and $f(x)=O\left(x^{k}\right)$ for some $k>0$ as $x \rightarrow \infty$, then $S_{N}(f)(x)$ converges to $f(x)$ uniformly on any compact subset of $[0, \infty)$, i.e., he generalized the Bernstein polynomials defined on $[0,1]$ to the infinite interval.

In section 3, our first observations are Lemmas 3.1 and 3.3. $S_{N}(f)(x)$ may be rewritten in terms of the Bergman kernel for the Bargmann-Fock space. This connection allows us to generalize the Szasz analytic function to any noncompact Kähler toric manifold. As an application of these observations, we will get a nice asymptotics of the classical Szasz analytic function $S_{h_{B F}^{N}}(f)$. If we integrate on both sides of this asymptotics, we will get Dedekind-Riemann sums over the orthant $\mathbb{R}_{+}^{m}$.

Before we define the generalized Szasz analytic functions, let's first introduce some notations (see sections 2 and 4 ). Let $(M, \omega)$ be a complex $m$-dimensional noncompact complete Kähler toric manifold with a proper moment map $\mu: M \rightarrow P$ with respected to the Hamiltonian $\mathbb{T}^{m}$ action and $\omega$, where $P$ is the image of the moment map, and denote $u$ as the

Date: December 2, 2018. 
symplectic potential on $P$. Let $(L, h)$ be a $\mathbb{T}^{m}$-invariant line bundle over $M$ with the Hermitian metric $h$. Denote $h^{N}$ as the Hermitian metric on the line bundle $L^{N}$ induced by $h$. Let $B_{h^{N}}: L^{2}\left(M, L^{N}\right) \rightarrow H_{L^{2}}^{0}\left(M, L^{N}\right)$ be the Bergman kernel which is an orthogonal projection. Then we have,

Definition: Assume $f \in C_{c}^{\infty}\left(\mathbb{R}^{m}\right)$ has compact support, then the generalized Szasz analytic function of $f(x)$ is defined by:

$$
\begin{gathered}
S_{h^{N}}(f)(x)=\frac{1}{B_{h^{N}}(z, z)} \mathcal{N}_{h^{N}} f(x) \\
\mathcal{N}_{h^{N}} f(x)=\sum_{\alpha \in \mathbb{Z}^{m} \cap P} f\left(\frac{\alpha}{N}\right) \frac{e^{N\left(u(x)+\left\langle\frac{\alpha}{N}-x, \nabla u(x)\right\rangle\right)}}{\left\|z^{\alpha}\right\|_{h^{N}}^{2}}
\end{gathered}
$$

where $z$ and $x$ are related by the moment map $z=\mu^{-1}(x),\left\{z^{\alpha}\right\}$ is a basis of $H_{L^{2}}^{0}\left(M, L^{N}\right)$ and $\alpha=\left(\alpha_{1}, \ldots, \alpha_{m}\right) \in \mathbb{Z}^{m} \cap P$ is a multi-index.

This generalized Szasz analytic function is analogous to the generalized Bernstein polynomials defined on any compact Kähler toric manifolds [Z1], and it has analogous properties and applications. In section 4, we first have the following proposition:

Proposition 1.1.

$$
S_{h^{N}}(f)(x)=\left.\frac{1}{B_{h^{N}}(z, z)} f\left(N^{-1} D_{\theta}\right) B_{h^{N}}\left(e^{i \theta} z, z\right)\right|_{\theta=0, z=\mu^{-1}(x)}
$$

where $D_{\theta}=\left(\frac{1}{i} \frac{\partial}{\partial \theta_{1}}, \ldots, \frac{1}{i} \frac{\partial}{\partial \theta_{m}}\right),\left\{\frac{1}{i} \frac{\partial}{\partial \theta_{k}}, k=1, \ldots, m\right\}$ are generators of $\mathbb{T}^{m}$ action on $M$.

By proposition 1.1 and the complete asymptotic expansion of the Bergman kernel for noncompact Kähler toric manifolds (Theorem 4.1 which we sketch the proof), we can prove the complete asymptotic expansion of our generalized Szasz functions which is one of our main results :

TheOREM 1.2. Let $(L, h) \rightarrow(M, \omega)$ be a $\mathbb{T}^{m}$-invariant Hermitian line bundle over a noncompact complete Kähler toric manifold with a proper moment map. Let $P$ be the moment polyhedral set, $S_{h^{N}}(f)(x)$ be the generalized Szasz analytic function of $f$ with compact support. If there exists $\gamma>0$, such that $\operatorname{Ric}(h)>\gamma(\omega,-\operatorname{Ric}(\omega))$, then:

$$
S_{h^{N}}(f)(x)=f(x)+\mathcal{L}_{1} f(x) N^{-1}+\mathcal{L}_{2} f(x) N^{-2}+\cdots+\mathcal{L}_{m} f(x) N^{-m}+O\left(N^{-m-1}\right)
$$

for $N$ large enough, where $\mathcal{L}_{j}$ is a differential operator of order $2 j$ depending only on the curvature of the metric $h$; the expansion may be differentiated infinitely many times.

Let's pick up a vertex of $P$, i.e., the fixed point of the torus $\mathbb{T}^{m}$ action, assume it to be 0 by choosing a new coordinate. If we use the dilation operator: $D_{\rho} f(x)=f(\rho x)$ for any real number $\rho$, we will straighten out $P$ so that the neighborhood of the fixed point becomes an orthant $\mathbb{R}_{+}^{m}$. If we dilate the Szasz analytic functions at the same time, the following theorem shows that $S_{h_{B F}^{1}}$ is a universal scaling limit. More generally, we can consider dilations near some facets of $P$, i.e., fixed by $\mathbb{T}^{k}, k<m$ action. In this case, we have to use the operation $\hat{D}_{\rho} f\left(x^{\prime}, x^{\prime \prime}\right)=f\left(\rho x^{\prime}, x^{\prime \prime}\right)$, i.e., dilate the first few coordinates and fix the left, then the similar result is valid. In fact, we will see that this theorem also generalizes the Poisson Limit Theorem to any toric Kähler manifold other than $\mathbb{C P}^{m}$. 
Theorem 1.3. Assume $f \in C_{c}^{\infty}\left(\mathbb{R}^{m}\right)$, for any $x \in P$, we have:

$$
\left(D_{\frac{1}{N}} S_{h^{N}} D_{\frac{1}{N}}^{-1}\right) f(x)=S_{h_{B F}^{1}}(f)(x)+b_{1}(x) N^{-1}+b_{2}(x) N^{-2}+\cdots
$$

$b_{j}(x)$ are all computable functions and $b_{1}(x)=\left[\frac{1}{2}\left\langle\left(M(x)-H_{z}\right) D_{x}, D_{x}\right\rangle+a_{1}(0)\right] S_{h_{B F}^{1}}(f)(x)$, where $M(x)=\operatorname{diag}\left\{x_{1}, x_{2}, \ldots, x_{m}\right\}, H_{z}$ is the Hessian matrix of the Kähler potential $\varphi$ and $a_{1}(z)$ is the scalar curvature of $\omega$. If we consider the dilate operator $\hat{D}_{\rho}$, we have,

$$
\left(\hat{D}_{\frac{1}{N}} S_{h^{N}} \hat{D}_{\frac{1}{N}}^{-1}\right) f\left(x^{\prime}, x^{\prime \prime}\right)=S_{h_{B F}^{1}}\left(f_{x^{\prime \prime}}\right)\left(x^{\prime}\right)+c_{1}\left(x^{\prime}, x^{\prime \prime}\right) N^{-1}+c_{2}\left(x^{\prime}, x^{\prime \prime}\right) N^{-2}+\cdots
$$

Here, $f_{x^{\prime \prime}}\left(x^{\prime}\right)$ means we fix the variables $x^{\prime \prime}$ and consider $f\left(x^{\prime}, x^{\prime \prime}\right)$ as a function of $x^{\prime}$, but $c_{j}\left(x^{\prime}, x^{\prime \prime}\right)$ are all computable functions of variables of $x^{\prime}$ and $x^{\prime \prime}$.

As an example, if we take our Kähler toric manifold as $\mathbb{C P}^{1}$, then the generalized Szasz analytic function $S_{h_{F S}^{N}}(f)(x)$ becomes the classical Bernstein polynomial. Hence, if $f$ is a smooth function on $[0, \infty)$ with compact support, then,

$$
\sum_{k=0}^{N}\left(\begin{array}{c}
N \\
k
\end{array}\right) f(k)\left(\frac{x}{N}\right)^{k}\left(1-\frac{x}{N}\right)^{N-k}=\sum_{k=0}^{\infty} e^{-x} \frac{x^{k}}{k !} f(k)+b_{1}(x) N^{-1}+b_{2}(x) N^{-2}+\cdots
$$

for $N$ large enough. In this Fubini-Study case, the Hessian matrix is $H_{z}=x(1-x)$ and the scalar curvature is a constant $S=1$; thus $a_{1}=S=1$. Hence, $b_{1}$ is given by $\frac{1}{2} x^{2} S_{h_{B F}^{1}}\left(f^{\prime \prime}\right)(x)+$ $S_{h_{B F}^{1}}(f)(x)=\frac{1}{2} x^{2}\left(\sum_{k=0}^{\infty} e^{-x} \frac{x^{k}}{k !} f^{\prime \prime}(k)\right)+\sum_{k=0}^{\infty} e^{-x} \frac{x^{k}}{k !} f(k)$. Moreover, equation (5) refines the Poisson Limit Theorem which says that $\left(\begin{array}{c}N \\ k\end{array}\right)\left(1-\frac{x}{N}\right)^{N-k}\left(\frac{x}{N}\right)^{k}$ converges to $e^{-x} x^{k} / k$ ! for $N$ large enough $\mathrm{Re}$.

As another example, if we dilate the generalized Szsaz analytic function for the unit Disc with the Poincaré metric, see equation (48), for $N \geq 2$, we have,

$$
\frac{N-1}{N+1} \cdot\left(1+\frac{x}{N}\right)^{-N} \sum_{k=0}^{\infty}(N)_{k} \frac{f(k)}{k !}\left(\frac{\frac{x}{N}}{1+\frac{x}{N}}\right)^{k}=\sum_{k=0}^{\infty} e^{-x} \frac{x^{k}}{k !} f(k)+c_{1}(x) N^{-1}+\cdots
$$

In this case, it's easy to compute the Hessian matrix is $H_{z}=x(1+x)$ and the scalar curvature is a constant $S=-1$. Hence, $c_{1}(x)=-\frac{1}{2} x^{2}\left(\sum_{k=0}^{\infty} e^{-x} \frac{x^{k}}{k !} f^{\prime \prime}(k)\right)-\sum_{k=0}^{\infty} e^{-x} \frac{x^{k}}{k !} f(k)$.

In section 5, we will compute the generalized Szasz analytic function for the unit ball $B^{m}$ with the Bergman metric and for the Kepler manifolds with an incomplete Kähler metric. In the case of the unit ball with the Bergman metric, we will see that the generalized Szasz analytic function relates this Bergman metric to the negative binomial distribution in the same sense that the Fubini-Study metric is related to the binomial distribution [Z1].

Before we prove our main results, some basic properties and examples about the Kähler toric manifolds are given in section 2 .

\section{KÄHLER TORIC MANIFOLDS}

In this section, we will give the definition of the noncompact complete Kähler toric manifold, discuss several basic properties and give two examples. Through the article, we use the following definition: 
Definition: A complex m-dimensional Kähler toric manifold is a complete Kähler manifold $(M, \omega)$ equipped with an effective Hamiltonian holomorphic real torus $\mathbb{T}^{m}=\mathbb{R}^{m} / 2 \pi \mathbb{Z}^{m}$ action and with a corresponding moment map $\mu: M \longrightarrow \mathbb{R}^{m}$.

As we can see from this definition, the study of the Kähler toric manifolds involves both the complex and symplectic structures. This definition is different from the one in the algebraic or complex geometry $[\mathrm{Bo}]$ :

Definition: An algebro-geometric toric variety is a (normal) irreducible variety $X$ equipped with an algebraic torus $\left(\mathbb{C}^{*}\right)^{m}$ action and containing $\left(\mathbb{C}^{*}\right)^{m}$ as an open dense orbit.

Regarding these two definitions, if the $\mathbb{T}^{m}$ action on the Kähler toric manifold $(M, \omega)$ doesn't extend to a $\left(\mathbb{C}^{*}\right)^{m}$ action, then by the second definition, $M$ is not an algebrogeometric toric variety. For example, in the Kähler toric world, the complex plane $\mathbb{C}$ equipped with the Euclidean metric and the unit disc equipped with the Bergman metric are both noncompact complete Kähler toric manifolds. But the unit disc is not an algebro-geometric toric variety since it is not invariant under the $\mathbb{C}^{*}$ action.

Next, let's recall some fundamental properties of Kähler toric manifolds [A1, A2, G, Z1]. Let $(M, \omega)$ be a complete toric Kähler manifold. Since the Kähler form $w=\partial \bar{\partial} \varphi$ (locally) is invariant under the real $\mathbb{T}^{m}$ action, then the Kähler potential $\varphi$ must be in the form of $\varphi(z)=F\left(|z|^{2}\right)$, where $F$ is a strictly convex function on $\mathbb{R}^{m}$ because of the positivity of $\omega$. From now on, let's write $z=e^{\frac{\rho}{2}+i \theta}$, then:

$$
\iota_{\frac{\partial}{\partial \theta_{j}}} \omega=d \frac{\partial \varphi}{\partial \rho_{j}}
$$

where $\frac{\partial}{\partial \theta_{j}}$ are generators of the $\mathbb{T}^{m}$ action. Hence, by the definition, the moment map $\mu: M \rightarrow \mathbb{R}^{m}$ with respect to $\omega$ and $\mathbb{T}^{m}$ action is given by:

$$
\mu\left(z_{1}, \ldots, z_{m}\right)=\nabla_{\rho} \varphi(z)=\nabla_{\rho} F\left(e^{\rho_{1}}, \ldots, e^{\rho_{m}}\right),
$$

The image of the moment map is called the moment polytope (compact) or moment polyhedral set(noncompact).

In the compact case, Delzant's Theorem [Del] says that the moment image of a compact symplectic toric manifold is a Delzant polytope, and every Delzant polytope is the moment image of a compact symplectic toric manifold. Moreover, two symplectic toric manifolds with the same Delzant polytope are equivariantly symplectomorphic. Here, the Delzant's polytope or polyhedral set is defined by:

$$
P=\left\{x \in\left(\mathbb{R}^{m}\right)^{*} \mid\left\langle x, v_{i}\right\rangle \leq \lambda_{i}, 1 \leq i \leq d\right\}
$$

where $d$ is the number of facets, $v_{1}, \ldots, v_{d} \in \mathbb{Z}^{m}$ is a set of primitive outward-pointing normal vectors to the facets and $\left(\mathbb{R}^{m}\right)^{*}$ is the dual of $\mathbb{R}^{m}$, see $\mathrm{CdS}$ (p.175-177) for more details.

In the noncompact case, if $(M, \omega)$ is a noncompact complete Kähler toric manifold with a proper moment map $\mu$, then the moment image is also a convex polyhedral set $P$ (noncompact) defined by (8). Here, a map is proper if the preimage of every compact set is compact. This properness is assumed through the article, otherwise, the moment image may be not convex $[\mathrm{P}]$. Furthermore, we can construct some noncompact complete Kähler toric manifolds from some special polyhedral sets following Delzant's construction [CdS, Del]. We will give an example in subsection 2.1.1 to see how it works. 
Finally, the symplectic potential $u$ associated to the Kähler potential is defined to be the Legendre dual of the Kähler potential $\varphi$ :

$$
u\left(x_{1}, \ldots, x_{m}\right)=\langle x, \rho\rangle-\varphi(\rho), x=\nabla_{\rho} \varphi
$$

Regarding this symplectic potential, see Theorem 2.8 in [A2], we have :

$$
u(x)=\sum_{i=1}^{d} \ell_{i}(x) \log \ell_{i}(x)+h(x)
$$

where $\ell_{i}=\lambda_{i}-\left\langle x, v_{i}\right\rangle, h \in C^{\infty}(P)$ and $u$ is smooth on the interior of $P$. Moreover, $G=\operatorname{Hess}_{x}(u)$ is positive definite on the interior of $P$.

2.1. Examples. In this section, we will study some examples about the noncompact toric manifolds. They come in three types: the total spaces of line bundle over some compact toric manifolds; the toric Sasaki-Kähler manifolds which is a metric cone over some odd dimensional Riemannian manifolds and the Reinhardt domains.

2.1.1. $\mathcal{O}(-k) \longrightarrow \mathbb{C P}^{1}$. Before we study the general case, let's consider $\mathcal{O}(-1) \rightarrow \mathbb{C P}^{1}$ first. This space is a Kähler toric manifold constructed by blowing-up one point over $\mathbb{C}^{2}$. More toric geometrical facts are given in [Bo, INOV] and also studied in [Le] by the method of symplectic cuts. First, we claim that: $\mathcal{O}(-1) \rightarrow \mathbb{C P}^{1}$ can be constructed by Delzant's method from the polyhedral set:

$$
\left\{(x, y) \in \mathbb{R}^{2} \mid x \geq 0, y \geq 0, x+y \geq 1\right\}
$$

Proof. Delzant's construction for the compact case can be found in [CdS] (p.24-30), in our proof, we will use the same notations as hers. In Delzant's setting, $n=2, d=3$, and $v_{1}=(-1,0), v_{2}=(0,-1), v_{3}=(-1,-1)$ are standard outward-pointing vectors, then we can describe the above polyhedral set as $\left\langle x, v_{i}\right\rangle \leq \lambda_{i}$, where $\lambda_{1}=\lambda_{2}=0$ and $\lambda_{3}=-1$. Now make the following projection:

$$
\pi: \mathbb{R}^{3} \longrightarrow \mathbb{R}^{2}
$$

which is defined by $\pi\left(e_{i}\right)=v_{i}$, where $e_{i}$ are standard basis of $\mathbb{R}^{3}$. Then the kernel of $\pi$ is spanned by $e_{1}+e_{2}-e_{3}$. Furthermore, $\pi$ induces a map $\mathbb{T}^{3} \longrightarrow \mathbb{T}^{2}$. If we denote $N$ as the kernel of this induced map, then $N$ can be described by $\left(e^{i \theta}, e^{i \theta}, e^{-i \theta}\right)$. Denote by $\mathfrak{n}$ the Lie algebra of $N$, then we have the following exact sequence:

$$
0 \rightarrow \mathfrak{n} \stackrel{i}{\rightarrow} \mathbb{R}^{3} \stackrel{\pi}{\rightarrow} \mathbb{R}^{2} \rightarrow 0
$$

where $i(x)=(x, x,-x), \pi(x, y, z)=(-x-z,-y-z)$. Then we have the dual exact sequence:

$$
0 \rightarrow\left(\mathbb{R}^{2}\right)^{*} \stackrel{\pi^{*}}{\rightarrow}\left(\mathbb{R}^{3}\right)^{*} \stackrel{i^{*}}{\rightarrow} \mathfrak{n}^{*} \rightarrow 0
$$

By a simple computation, we have $i^{*}(x, y, z)=x+y-z$. Denote by $\mu$ the moment map for the natural Hamiltonian $\mathbb{T}^{3}$ action on $\mathbb{C}^{3}$, i.e.,

$$
\mu: \mathbb{C}^{3} \longrightarrow\left(\mathbb{R}^{3}\right)^{*}, \quad\left(z_{1}, z_{2}, z_{3}\right) \rightarrow\left(-\left|z_{1}\right|^{2},-\left|z_{2}\right|^{2},-\left|z_{3}\right|^{2}-1\right)
$$

In addition, the subtorus $N=\left(e^{i \theta}, e^{i \theta}, e^{-i \theta}\right) \subset \mathbb{T}^{3}$ also acts on $\mathbb{C}^{3}$ in a natural way:

$$
\left(e^{i \theta}, e^{i \theta}, e^{-i \theta}\right) \cdot\left(z_{1}, z_{2}, z_{3}\right)=\left(e^{i \theta} z_{1}, e^{i \theta} z_{2}, e^{-i \theta} z_{3}\right)
$$


In fact, this action is the restriction of $\mathbb{T}^{3}$ action and is Hamiltonian with the moment map:

$$
\left(i^{*} \circ \mu\right)\left(z_{1}, z_{2}, z_{3}\right)=-\left|z_{1}\right|^{2}-\left|z_{2}\right|^{2}+\left|z_{3}\right|^{2}+1
$$

Then the zero-level set of this moment map is:

$$
\left(i^{*} \circ \mu\right)^{-1}(0)=\left\{\left(z_{1}, z_{2}, z_{3}\right) \in \mathbb{C}^{3}:\left|z_{1}\right|^{2}+\left|z_{2}\right|^{2}-\left|z_{3}\right|^{2}=1\right\}
$$

Hence the reduced space is :

$$
\left(i^{*} \circ \mu\right)^{-1}(0) / N=\mathcal{O}(-1)
$$

which completes the proof. For the last equality, see [Bo](p.49) for more details.

2.1.2. Toric Sasaki-Kähler manifolds. Let $(M, g)$ be a real $(2 m-1)$-dimensional Riemannian manifold, $M$ is called Sasaki-Kähler if the cone $\left(M \times \mathbb{R}_{+}, d s^{2}\right)$ is a noncompact complex $m$-dimensional Kähler manifold, here $d s^{2}=d r^{2}+r^{2} g$ and $d s^{2}$ is incomplete at $r=0$. In particular, $M$ is called toric Sasaki-Kähler if $M \times \mathbb{R}_{+}$is a toric Kähler manifold, $M$ is called Sasaki-Einstein if $d s^{2}$ is a Ricci-flat Kähler metric.

As an example, consider the Conifold $X$ which is a complex 3-dimensional affine variety defined by a single equation in $\mathbb{C}^{4}$ :

$$
z_{1} z_{2}-z_{3} z_{4}=0
$$

By Delzant's method again, $X$ can be constructed by four lattice vectors in $\mathbb{Z}^{3}$ :

$$
v_{1}=(1,1,1), v_{2}=(1,0,1), v_{3}=(0,1,1), v_{4}=(0,0,1)
$$

If we take $m=(0,0,1)$, then these four lattice vectors are in the same hyperplane $\left\langle v_{i}, m\right\rangle=1$, hence $X$ is toric Calabi-Yau variety [Bo]. One interesting fact about $X$ is that: $X$ is a cone of the Sasaki-Einstein manifold $\left(S^{2} \times S^{3}, g\right), g$ is a homogeneous metric defined by [CO]:

$$
g=\frac{1}{2}\left(d \theta_{1}^{2}+\sin ^{2} \theta_{1} d \varphi_{1}^{2}+d \theta_{2}^{2}+\sin ^{2} \theta_{2} d \varphi_{2}^{2}\right)+\frac{1}{3}\left(d \psi+\cos \theta_{1} d \varphi_{1}+\cos \theta_{2} d \varphi_{2}\right)^{2}
$$

Here $\theta_{i}, \varphi_{i}, i=1,2$, are usual polar and axial coordinates on two round two-spheres, and $\psi$ is a coordinate on a principle $U(1)$ bundle over $S^{2} \times S^{2}$. Here $\psi$ has period $4 \pi$ so that the Chern numbers over the two two-spheres are both equal to one. Then the Kähler form on $X$ is [MS]:

$$
\omega=\frac{1}{2} r^{2}\left(\sin \theta_{1} d \theta_{1} \wedge d \varphi_{1}+\sin \theta_{2} d \theta_{2} \wedge d \varphi_{2}\right)-r d r \wedge\left(d \psi+\cos \theta_{1} d \varphi_{1}+\cos \theta_{2} d \varphi_{2}\right)
$$

Defining $2 \nu=\psi$, so that $\nu$ has period $2 \pi$, then we have the following basis for $\mathbb{T}^{3}$ action:

$$
e_{1}=\frac{\partial}{\partial \varphi_{1}}+\frac{1}{2} \frac{\partial}{\partial \nu}, e_{2}=\frac{\partial}{\partial \varphi_{2}}+\frac{1}{2} \frac{\partial}{\partial \nu}, e_{3}=\frac{\partial}{\partial \nu}
$$

Then the corresponding moment map is:

$$
\mu=\left(\frac{1}{2} r^{2}\left(\cos \theta_{1}+1\right), \frac{1}{2} r^{2}\left(\cos \theta_{2}+1\right), r^{2}\right)
$$

Now, let's change variables, $X$ can be rewritten as:

$$
z_{1}^{2}+z_{2}^{2}+z_{3}^{2}+z_{4}^{2}=0
$$

Hence, $X-\{0\}$ will be a 3 -dimensional Kepler manifold, we will consider the generalized Szasz analytic function for this Conifold $X$ in the subsection 5.2. 
2.1.3. Reinhardt Domains. Let's recall that an open set $X \subset \mathbb{C}^{m}$ is a Reinhardt domain if $\left(z_{1}, \ldots, z_{m}\right) \in X$ implies that $\left(e^{i \theta_{1}} z_{1}, \ldots, e^{i \theta_{m}} z_{m}\right) \in X$, i.e., it's closed under the natural torus $\mathbb{T}^{m}$ action.

Now claim: any bounded pseudoconvex Reinhardt domains with $C^{1}$-smooth boundary is a Kähler toric manifold. First, for any bounded domain in $\mathbb{C}^{m}$, there exists a canonical Kähler metric: the Bergman metric $\varphi_{B}$. Moreover, $\varphi_{B}$ is invariant under any holomorphic automorphism. Hence, for any bounded pseudoconvex Reinhardt domain, $\varphi_{B}$ is invariant under $\mathbb{T}^{m}$ action, i.e., $\varphi_{B}\left(z_{1}, \ldots, z_{m}\right)=\varphi_{B}\left(\left|z_{1}\right|^{2}, \ldots,\left|z_{m}\right|^{2}\right)$. In addition, $\varphi_{B}$ is complete for any pseudoconvex domain with $C^{1}$-smooth boundary[DO]. Thus by equation (6), this $\mathbb{T}^{m}$ action is actually Hamiltonian with the moment map $\mu\left(z_{1}, \ldots, z_{m}\right)=\nabla_{\rho} \varphi_{B}(z)$. At last, $\mathbb{T}^{m}$ acts on $X$ by multiplication, thus this action is effective. Hence, the claims follows by the definition of Kähler toric manifolds.

Now, let's consider the Reinhardt domain $\Omega_{p}=\left\{\left.(z, w) \in \mathbb{C}^{2}|| z\right|^{2}+|w|^{2 p}<1, p \geq 1\right\}$. This domain is a bounded pseudoconvex Reinhardt domain with the complete Bergman metric, hence $\Omega_{p}$ has a toric structure. Regarding this domain, we claim: the polyhedral set for the Kähler toric manifold $\Omega_{p}$ is:

$$
\left\{(x, y) \in \mathbb{R}^{2} \mid x \geq 0, \frac{y}{p} \geq 0\right\}
$$

In fact, this is a "labeled polyhedral set"[LS].

Proof. We have to pick up any invariant complete Kähler metric over $\Omega_{p}$ and compute the corresponding symplectic potential $u$, then the facets of the polyhedral set can be seen because of identity (10). In fact, we can choose the complete Kähler metric defined by the defining function:

$$
\varphi_{p}=-\log \left(1-|z|^{2}-|w|^{2 p}\right)
$$

when $p \geq 1, \partial \bar{\partial} \varphi_{p}$ is a well-defined Kähler metric over $\Omega_{p}$, see [CY](p.509) for details and more general cases. Then the moment map is:

$$
\mu(z, w)=\left(\frac{|z|^{2}}{1-|z|^{2}-|w|^{2 p}}, \frac{p|w|^{2 p}}{1-|z|^{2}-|w|^{2 p}}\right)
$$

In addition, the symplectic potential given by the Legendre dual of $\varphi_{p}$ is:

$$
u(x, y)=x \log x+\frac{y}{p} \log \frac{y}{p}-\left(1+x+\frac{y}{p}\right) \log \left(1+x+\frac{y}{p}\right)
$$

Then two facets of the moment polyhedral set are given by $\ell_{1}(x, y)=x$ and $\ell_{2}(x, y)=\frac{y}{p}$. Thus, the moment polyhedral set must be $\left\{(x, y) \in \mathbb{R}^{2} \mid x \geq 0, \frac{y}{p} \geq 0\right\}$, since it's the image of the moment $\operatorname{map} \mu(z, w) \geq 0$.

Once we get the moment polyhedral set, we can construct special metrics on $\Omega_{p}$ by constructing some symplectic potentials. In fact, $\left(\Omega_{p}, \partial \bar{\partial} \varphi_{p}\right)$ is a Kähler manifold of constant scalar curvature for all $p$, where $\varphi_{p}$ is defined by equation (12). To show this, let's compute the inverse of the Hessian matrix of the symplectic potential $u$ (13):

$$
G^{-1}=\left(\begin{array}{cc}
(1+x) x & x y \\
x y & (p+y) y
\end{array}\right)
$$


Now, let's recall that the scalar curvature of a Kähler toric metric can be given in terms of the corresponding symplectic potential $u$ :

$$
S=-\sum_{i, j} \frac{\partial^{2} u^{i j}}{\partial x_{i} \partial x_{j}}
$$

where $u^{i j}$ is the matrix $G^{-1}$. This is the main result in [A1, and it will be automatically valid for noncompact Kähler toric manifolds since the scalar curvature is a local property. Hence, using this formula, we have: $S=-6$.

\section{Bargmann-Fock space and Classical Szasz Analytic Functions}

In this section, we will relate the classical Szasz analytic function with the Bargmann-Fock space. The Bargmann-Fock space is the space of entire functions on $\mathbb{C}^{m}$ which are $L^{2}$ integral with respect to the Bargmann-Fock metric, i.e., $\mathcal{H}^{2}\left(\mathbb{C}^{m}, \pi^{-m} e^{-N|z|^{2}} d z d \bar{z}\right)$. This space is a Hilbert space and it has a basis $\left\{z^{\alpha}\right\}$ with $\alpha \in \mathbb{Z}_{+}^{m}=\left\{\left(\alpha_{1}, \ldots, \alpha_{n}\right) \in \mathbb{Z}^{m}, \alpha_{j} \geq 0\right.$ for all $\left.j\right\}$. Since

$$
\left\|z^{\alpha}\right\|_{h_{B F}^{N}}^{2}=\int_{\mathbb{C}^{m}} z^{\alpha} \bar{z}^{\alpha} e^{-N|z|^{2}} \pi^{-m} d z d \bar{z}=\frac{1}{N^{m+|\alpha|}} \int_{\mathbb{C}^{m}} z^{\alpha} \bar{z}^{\alpha} e^{-|z|^{2}} \pi^{-m} d z d \bar{z}=\frac{\alpha !}{N^{m+|\alpha|}}
$$

So

$$
\left\{\frac{z^{\alpha}}{\sqrt{\frac{\alpha !}{N^{m+|\alpha|}}}}, \alpha \in \mathbb{Z}_{+}^{m}\right\}
$$

is a orthonormal basis of $\mathcal{H}^{2}\left(\mathbb{C}^{m}, \pi^{-m} e^{-N|z|^{2}} d z d \bar{z}\right)$. Now we can express the classical Szasz function in terms of Bergman kernel for the Bargmann-Fock space.

LEMma 3.1. If $f \in \mathbb{C}_{c}^{\infty}\left(\mathbb{R}_{+}^{m}\right)$ and $S_{N}(f)(x)$ is the classical Szasz analytic function (11). Then,

$$
S_{N}(f)(x)=\frac{1}{B_{h_{B F}^{N}}(z, z)} \sum_{\alpha \in \mathbb{Z}_{+}^{m}} f\left(\frac{\alpha}{N}\right) \frac{e^{N\left(u_{B F}(x)+\left\langle\frac{\alpha}{N}-x, \nabla u_{B F}(x)\right\rangle\right)}}{\left\|z^{\alpha}\right\|_{h_{B F}^{N}}^{2}},
$$

where $\mu_{B F}(z)=|z|^{2}$ is the moment map, $B_{h_{B F}^{N}}(z, z)$ is the Bergman kernel for the BargmannFock space, $x=\mu_{B F}(z)=|z|^{2}, u_{B F}(x)=x \log x-x$ is the symplectic potential over the orthant $\mathbb{R}_{+}^{m}=\left\{\left(x_{1}, \ldots, x_{m}\right) \in \mathbb{R}^{m}, x_{j} \geq 0\right.$ for all $\left.j\right\}$.

Proof. First, by identity (23) below, we have the Bergman kernel on the diagonal:

$$
\begin{aligned}
B_{h_{B F}^{N}}(z, z) & =\sum_{\alpha \in \mathbb{Z}_{+}^{m}} \frac{|z|^{2 \alpha}}{\left\|z^{\alpha}\right\|_{h_{B F}^{N}}^{2}} e^{-N|z|^{2}}=\sum_{\alpha \in \mathbb{Z}_{+}^{m}} \frac{|z|^{2 \alpha}}{\frac{\alpha !}{N^{m+|\alpha|}}} e^{-N|z|^{2}}=\sum_{n=0}^{\infty} \sum_{|\alpha|=n} \frac{|z|^{2 \alpha}}{\frac{\alpha !}{N^{m+|\alpha|}}} e^{-N|z|^{2}} \\
= & N^{m} e^{-N|z|^{2}} \sum_{n=0}^{\infty} \sum_{|\alpha|=n} \frac{|z|^{2 \alpha} N^{|\alpha|}}{\alpha !}=N^{m} e^{-N|z|^{2}} \sum_{n=0}^{\infty} \frac{N^{n}|z|^{n}}{n !}=N^{m}
\end{aligned}
$$

In the Bargmann-Fock case, the Kähler potential is:

$$
\varphi(z)=|z|^{2}=e^{\rho}, z=e^{\frac{\rho}{2}+i \theta}
$$

Hence, by equation(7), the moment map is given by:

$$
\mu_{B F}\left(z_{1}, \ldots, z_{m}\right)=\left(\left|z_{1}\right|^{2}, \ldots,\left|z_{m}\right|^{2}\right)
$$


In addition, the symplectic potential given by the Legendre transform of $\varphi(z)=|z|^{2}$ is:

$$
u(x)=\sum_{j=1}^{m} x_{j} \log x_{j}-\sum_{j=1}^{m} x_{j}
$$

Combining equations (15), (17) and (20), we have:

$$
\begin{gathered}
\frac{1}{B_{h_{B F}^{N}}(z, z)} \sum_{\alpha \in \mathbb{Z}_{+}^{m}} f\left(\frac{\alpha}{N}\right) \frac{e^{N\left(u_{B F}(x)+\left\langle\frac{\alpha}{N}-x, \nabla u_{B F}(x)\right\rangle\right)}}{\left\|z^{\alpha}\right\|_{h_{B F}^{N}}^{2}} \\
=N^{-m} \sum_{\alpha \in \mathbb{Z}_{+}^{m}} f\left(\frac{\alpha}{N}\right) \frac{e^{-N x}(N x)^{\alpha}}{\frac{\alpha !}{N^{m+|\alpha|}}}=e^{-N x} \sum_{\alpha \in \mathbb{Z}_{+}^{m}} \frac{(N x)^{\alpha}}{\alpha !} f\left(\frac{\alpha}{N}\right)=S_{N}(f)
\end{gathered}
$$

REMARK 3.2. In view of the moment map (19), it is easy to see that the moment polyhedral set for the Kähler toric manifold $\mathbb{C}^{m}$ with Euclidean metric is the orthant $\mathbb{R}_{+}^{m}$.

We now prove a special case of Proposition 1.1 for the classical Szasz analytic function :

LEMMA 3.3. With the same notations as above,

$$
S_{N}(f)(x)=\left.\frac{1}{B_{h_{B F}^{N}}(z, z)} f\left(N^{-1} D_{\theta}\right) B_{h_{B F}^{N}}\left(e^{i \theta} z, z\right)\right|_{\theta=0, z=\mu_{h_{B F}}^{-1}(x)}
$$

where $D_{\theta}=\left(\frac{1}{i} \frac{\partial}{\partial \theta_{1}}, \ldots, \frac{1}{i} \frac{\partial}{\partial \theta_{m}}\right),\left\{\frac{1}{i} \frac{\partial}{\partial \theta_{k}}, k=1, \ldots, m\right\}$ are generators of $\mathbb{T}^{m}$ action over $\mathbb{C}^{m}$.

Proof. The key to the proof is to get the Bergman kernel off the diagonal, by identity (23) again, in our Bargmann-Fock case,

$$
\begin{aligned}
B_{h_{B F}^{N}}\left(e^{i \theta} z, z\right) & =\sum_{\alpha \in \mathbb{Z}_{+}^{m}} e^{i\langle\alpha, \theta\rangle} \frac{|z|^{2 \alpha}}{\left\|z^{\alpha}\right\|_{h_{B F}^{N}}^{2}} e^{-N|z|^{2}}=e^{-N|z|^{2}} \sum_{\alpha \in \mathbb{Z}_{+}^{m}} e^{i\langle\alpha, \theta\rangle} \frac{|z|^{2 \alpha}}{\frac{\alpha !}{N^{m+|\alpha|}}} \\
& =N^{m} e^{-N x} \sum_{\alpha \in \mathbb{Z}_{+}^{m}} \frac{(N x)^{\alpha}}{\alpha !} e^{i\langle\theta, \alpha\rangle}, z=\mu_{h_{B F}}^{-1}(x)
\end{aligned}
$$

then the lemma follows by Spectral theorem (24) and the fact that $B_{h_{B F}^{N}}(z, z)=N^{m}$.

REMARK 3.4. Once we have Lemmas 3.1 and 3.3 which relate the classical Szasz analytic functions to $\left(\mathbb{C}^{m}, d z \wedge d \bar{z}\right)$, we can generalize this relation to any noncompact Kähler toric manifold $(M, \omega)$, hence Definition 1 is given immediately. Under this new definition, the classical Szasz analytic function $S_{N}(f)(x)$ is denoted as $S_{h_{B F}^{N}}(f)(x)$.

3.1. Application: Dedekind-Riemann sums over $\mathbb{R}_{+}^{m}$. In this section, we will first get a nice complete asymptotic of the classical Szasz analytic functions. Then if we integrate this complete asymptotics, we will get the Dedekind-Riemann sums over the orthant $\mathbb{R}_{+}^{m}$.

Lemma 3.5. For any $f \in C_{c}^{\infty}\left(\mathbb{R}_{+}^{m}\right)$, we have:

$$
S_{h_{B F}^{N}}(f)=e^{-N x} \sum_{\alpha=0}^{\infty} \frac{(N x)^{\alpha}}{\alpha !} f\left(\frac{\alpha}{N}\right)=\sum_{n=0}^{\infty} \frac{x^{n}}{n !} P^{n}\left(\frac{1}{i} \frac{\partial}{\partial x}\right) f(x) \bmod O\left(N^{-\infty}\right)
$$

where $\alpha \in \mathbb{Z}_{+}^{m}, P(x)=\sum_{j=2}^{\infty} \frac{(i x)^{j}}{N^{j-1} j !}$ and $P^{n}(x)$ means the nth power of $P(x)$. 
Proof. First by Fourier Transform, we can rewrite (21) as:

$$
S_{h_{B F}^{N}}(f)=\int_{\mathbb{R}^{m}} \hat{f}(\xi) e^{N\left(F\left(e^{i N^{-1}} \xi|z|^{2}\right)-F\left(|z|^{2}\right)\right)} d \xi
$$

where $F(x)=x$. Hence, use the Taylor expansion and Spectral theorem, we have:

$$
\begin{aligned}
& S_{h_{B F}^{N}}(f)=\int_{\mathbb{R}^{m}} \hat{f}(\xi) e^{N\left(e^{i N^{-1} \xi}|z|^{2}-|z|^{2}\right)}=\int_{\mathbb{R}^{m}} \hat{f}(\xi) e^{\langle i x, \xi\rangle} e^{N\left[\frac{\left(i N^{-1} \xi\right)^{2}}{2 !}+\frac{\left(i N^{-1} \xi\right)^{3}}{3 !} \ldots\right] x} \\
& =\int_{\mathbb{R}^{m}} \hat{f}(\xi) e^{\langle i x, \xi\rangle} e^{P(\xi) x}=\sum_{n=0}^{\infty} \frac{x^{n}}{n !} \int_{\mathbb{R}^{m}} \hat{f}(\xi) e^{\langle i x, \xi\rangle} P^{n}(\xi)=\sum_{n=0}^{\infty} \frac{x^{n}}{n !} P^{n}\left(\frac{1}{i} \frac{\partial}{\partial x}\right) f(x)
\end{aligned}
$$

where we have already used the fact $x=\mu(z)=|z|^{2}$.

THEOREM 3.6. Assume $f$ is a smooth function with compact support on $\mathbb{R}_{+}^{m}$, then:

$$
\frac{1}{N^{m}} \sum_{\alpha \in \mathbb{Z}_{+}^{m}} f\left(\frac{\alpha}{N}\right)=\int_{\mathbb{R}_{+}^{m}} f(x) d x-\frac{1}{2 N} \int_{\mathbb{R}_{+}^{m}} x f^{(2)}(x) d x+\frac{1}{N^{2}} \int_{\mathbb{R}_{+}^{m}}\left(\frac{x f^{(3)}}{3}+\frac{x^{2} f^{(4)}}{8}\right) d x+\ldots
$$

where $d x$ is Lebesgue measure. Furthermore, we can simplify the second term to be:

$$
-\frac{1}{2 N} \int_{\mathbb{R}_{+}^{m}} x f^{(2)}(x) d x=\frac{1}{2 N} \int_{\partial \mathbb{R}_{+}^{m}} f(x) d x
$$

Proof. Let's integrate on both sides of Proposition 3.5, by a simple computation, we have:

$$
\frac{1}{N^{m}} \sum_{\alpha \in \mathbb{Z}_{+}^{m}} f\left(\frac{\alpha}{N}\right)=\int_{\mathbb{R}_{+}^{m}} e^{-N x} \sum_{\alpha=0}^{\infty} \frac{(N x)^{\alpha}}{\alpha !} f\left(\frac{\alpha}{N}\right) d x=\int_{\mathbb{R}_{+}^{m}} \sum_{n=0}^{\infty} \frac{x^{n}}{n !} P^{n}\left(\frac{1}{i} \frac{\partial}{\partial x}\right) f(x) d x
$$

Hence, the complete asymptotics follows since we can expand $P^{n}\left(\frac{1}{i} \frac{\partial}{\partial x}\right)$ by iteration. The first three terms in the theorem are given if we compute $P^{n}\left(\frac{1}{i} \frac{\partial}{\partial x}\right)$ up to $n=2$.

\section{Generalized Szasz Analytic Expansion for any Kähler toric manifold}

In this section, we will first recall some basic properties about the Bergman kernel and then prove its complete asymptotics for the noncompact Kähler manifolds. Then we will prove Theorems 1.2 and 1.3 in the introduction.

4.1. Bergman kernel. Let's review the Bergman kernel on the compact Kähler manifolds first (see [Lu, [SZ, Z1, Z2]) and generalize it naturally to the noncompact case.

Let $L \rightarrow M$ be a positive holomorphic line bundle over a compact complex manifold. This means there exists a smooth Hermitian metric $h$ on $L$ with positive curvature form $\operatorname{Ric}(h)=-\partial \bar{\partial} \log \left\|e_{L}\right\|_{h}^{2}$, where $e_{L}$ is a local holomorphic frame of $L$ over an open set $U \subset M$, and $\left\|e_{L}\right\|_{h}^{2}=h\left(e_{L}, e_{L}\right)$ denotes the $h$-norm of $e_{L}$. The metric $h$ induces a Hermitian metric $h^{N}$ on $L^{N}$ by $\left\|e_{L}^{\otimes N}\right\|_{h^{N}}=\left\|e_{L}\right\|_{h}^{N}$. Furthermore, we can give $M$ a Kähler form $\omega=\frac{\sqrt{-1}}{2} \operatorname{Ric}(h)$ and the induced volume form $\omega_{m}=\omega^{m} / m$ !. If we write $\left\|e_{L}\right\|_{h}^{2}=e^{-\varphi}$ locally, then we have the following two identities

$$
\begin{aligned}
\omega & =\frac{\sqrt{-1}}{2} \partial \bar{\partial} \varphi \\
\left\|e_{L}^{\otimes N}\right\|_{h^{N}}^{2} & =\left\|e_{L}\right\|_{h}^{2 N}=e^{-N \varphi}
\end{aligned}
$$


Let $H^{0}\left(M, L^{N}\right)$ be the weighted space of all $L^{2}$ global holomorphic sections of the line bundle $L^{N}$. This means for each local frame $e_{L}$ of $L$, we can write each section of $H^{0}\left(M, L^{N}\right)$ as $s=f e_{L}^{\otimes N}$, where $f$ is a holomorphic function. Furthermore, $s$ satisfies:

$$
\|s\|_{h^{N}}^{2}=\int_{M}|f|^{2} e^{-N \varphi} \omega_{m}<\infty
$$

Thus $H^{0}\left(M, L^{N}\right)$ will be a Hilbert space with the natural inner product induced by $h^{N}$ :

$$
\left\langle s_{1}, s_{2}\right\rangle_{h^{N}}=\int_{M} h^{N}\left(s_{1}, s_{2}\right) \omega_{m}=\int_{M} f_{1} \bar{f}_{2} e^{-N \varphi} \omega_{m}
$$

where $s_{1}=f_{1} e_{L}^{\otimes N}, s_{2}=f_{2} e_{L}^{\otimes N} \in H^{0}\left(M, L^{N}\right)$.

We now define the Bergman kernels as the orthogonal projection from the $L^{2}$ integral sections to the holomorphic sections, i.e., $B_{h^{N}}: L^{2}\left(M, L^{N}\right) \rightarrow H^{0}\left(M, L^{N}\right)$. Furthermore, if $\left\{s_{j}^{N}\right\}_{j=1}^{d_{N}}$ is an orthonormal basis of $H^{0}\left(M, L^{N}\right)$, then:

$$
B_{h^{N}}(z, w)=\sum_{j=1}^{d_{N}} s_{j}^{N}(z) \otimes \overline{s_{j}^{N}(w)}
$$

where $d_{N}=\operatorname{dim} H^{0}\left(M, L^{N}\right)$.

When we consider noncompact Kähler manifolds, denote $H_{L^{2}}^{0}\left(M, L^{N}\right)$ as the weighted space of all $L^{2}$ global holomorphic sections of the line bundle $L^{N}$, then $H_{L^{2}}^{0}\left(M, L^{N}\right)$ becomes an infinite Hilbert space, there are infinite orthogonal basis $\left\{s_{j}^{N}\right\}_{j=1}^{\infty}$. But we can still define the Bergman kernel as the orthogonal projection i.e., $B_{h^{N}}: L^{2}\left(M, L^{N}\right) \rightarrow H_{L^{2}}^{0}\left(M, L^{N}\right)$.

Now let's turn to the proof of Proposition 1.1.

Proof. The monomials $\left\{z^{\alpha}\right\}$ with $\alpha \in \mathbb{Z}^{m} \cap P$ form a basis of $H_{L^{2}}^{0}\left(M, L^{N}\right)$ [NOV, [F, thus we have the Bergman Kernel off the diagonal by identity (23):

$$
B_{h^{N}}\left(e^{i \theta} z, z\right)=\sum_{\alpha \in \mathbb{Z}^{m} \cap P} \frac{\left|z^{\alpha}\right|^{2} e^{-N \varphi(z)} e^{i\langle\alpha, \theta\rangle}}{\left\|z^{\alpha}\right\|_{h^{N}}^{2}}
$$

By Spectral theorem, we have the general formula for any smooth function $\psi$ :

$$
\left.e^{i\left\langle\xi, N^{-1} D_{\theta}\right\rangle} \psi\left(e^{i \theta} \omega\right)\right|_{\theta=0}=\psi\left(e^{i \frac{\xi}{N}} \omega\right)
$$

Then by this formula and the fact that $\varphi(z)=F\left(|z|^{2}\right)$, we have:

$$
\left.e^{i\left\langle\xi, N^{-1} D_{\theta}\right\rangle} B_{h^{N}}\left(e^{i \theta} z, z\right)\right|_{\theta=0}=\sum_{\alpha \in \mathbb{Z}^{m} \cap P} \frac{e^{i\left\langle N^{-1} \alpha, \xi\right\rangle}\left|z^{\alpha}\right|^{2} e^{-N F\left(|z|^{2}\right)}}{\left\|z^{\alpha}\right\|_{h^{N}}^{2}}
$$

Next, given $f \in C^{\infty}\left(\mathbb{R}^{m}\right)$, we can define $f\left(D_{\theta}\right)$ by Fourier transformation:

$$
f\left(D_{\theta}\right)=\int_{\mathbb{R}^{m}} \hat{f}(\xi) e^{i\left\langle\xi, D_{\theta}\right\rangle} d \xi
$$

Now apply $f\left(N^{-1} D_{\theta}\right)$ on both sides of (25):

$$
\left.f\left(N^{-1} D_{\theta}\right) B_{h^{N}}\left(e^{i \theta} z, z\right)\right|_{\theta=0}=\left.\int_{\mathbb{R}^{m}} \hat{f}(\xi) e^{i\left\langle\xi, N^{-1} D_{\theta}\right\rangle} B_{h^{N}}\left(e^{i \theta} z, z\right)\right|_{\theta=0} d \xi
$$




$$
\begin{gathered}
=\sum_{\alpha \in \mathbb{Z}^{m} \cap P}\left[\frac{\left|z^{\alpha}\right|^{2} e^{-N F\left(|z|^{2}\right)}}{\left\|z^{\alpha}\right\|_{h^{N}}^{2}} \int_{\mathbb{R}^{m}} \hat{f}(\xi) e^{i\left\langle N^{-1} \alpha, \xi\right\rangle} d \xi\right]=\sum_{\alpha \in \mathbb{Z}^{m} \cap P} f\left(\frac{\alpha}{N}\right) \frac{\left|z^{\alpha}\right|^{2} e^{-N F\left(|z|^{2}\right)}}{\left\|z^{\alpha}\right\|_{h^{N}}^{2}} \\
=\sum_{\alpha \in \mathbb{Z}^{m} \cap P} f\left(\frac{\alpha}{N}\right) \frac{e^{N\left(u(x)+\left\langle\frac{\alpha}{N}-x, \nabla u(x)\right\rangle\right)}}{\left\|z^{\alpha}\right\|_{h^{N}}^{2}}=\mathcal{N}_{h^{N}} f(x)
\end{gathered}
$$

In the next to the last equality, we have to plug in formula (9). Hence, the proposition follows by dividing $B_{h^{N}}(z, z)$.

\subsection{Complete asymptotics of Bergman kernel for noncompact Kähler manifolds.}

Now, let's consider the complete asymptotics of Bergman kernel for any noncompact Kähler manifold. In the compact case, the classical result is Tian-Yau-Zelditch Theorem [Lu, T, ZZ2]. A very nice proof is given in [BBS]. For the noncompact case, we have:

THEOREM 4.1. Let $(M, \omega)$ be a complex m-dimensional noncompact complete Kähler manifold, and let $(L, h) \rightarrow(M, \omega)$ be a positive Hermitian holomorphic line bundle. Let $g$ be a Kähler metric on $M$ corresponding to the Kähler form $\omega=\operatorname{Ric}(h)$. If there exists $\gamma>0$, such that $\operatorname{Ric}(h)>-\gamma \operatorname{Ric}(g)$, then on any compact subset $K \subset M$, we have:

$$
B_{h^{N}}(z, z)=\sum_{i=0}^{\infty}\left\|s_{i}^{N}(z)\right\|_{h^{N}}^{2}=N^{m}\left(1+a_{1}(z) N^{-1}+a_{2}(z) N^{-2}+\cdots\right)
$$

where $a_{j}(z)$ are smooth on $K$ and $a_{1}(z)$ is the scalar curvature of $\omega$.

In particular, for the Kähler toric manifold with the Kähler potential $F\left(|z|^{2}\right)$, we have:

$$
B_{h^{N}}(z, w)=e^{N\left(F(z \cdot \bar{w})-\frac{1}{2}\left(F\left(|z|^{2}\right)+F\left(|w|^{2}\right)\right)\right)} A_{N}(z, w) \bmod N^{-\infty}
$$

where $F(z \cdot \bar{w})$ is the almost analytic extension of $F\left(|z|^{2}\right)$ and $A_{N}(z, w)=N^{m}\left(1+a_{1}(z, w) N^{-1}+\right.$ ...) a semi-classical symbol of order $m$.

Sketch of the proof: The proof follows the one in [BBS] with a few modifications. We fix our small coordinate neighborhood to be the unit ball $B$ of $\mathbb{C}^{m}, u$ is a holomorphic function on $B$, we can define the local expression of the norm of a section of $L^{N}$ over $B$ as:

$$
\|u\|_{h^{N}, l o c}^{2}=\int_{B}|u|^{2} e^{-N \varphi} \omega_{m}
$$

In [BBS], they first have their Proposition 2.7: there exists a local asymptotic Bergman kernel:

$$
B_{h^{N}, l o c}^{k}=N^{m}\left(1+a_{1}(z, \bar{w}) N^{-1}+\ldots+a_{k}(z, \bar{w}) N^{-k}\right)
$$

where $a_{j}(z, \bar{w})$ are defined in a fixed neighborhood of $z$ explicitly given and $a_{1}(z, \bar{z})$ is the scalar curvature of $\omega$, such that:

$$
u(x)=\left\langle\chi u, B_{h^{N}, l o c}^{k}\right\rangle_{h^{N}, l o c}+O\left(N^{-k}\right)\|u\|_{h^{N}, l o c}
$$

where $\chi$ is a smooth function supported in the unit ball $B$ and equal to one on the ball of radius $1 / 2$. Since this estimate is a local property, it will be automatically true for the noncompact Kähler manifold.

Next, globally, they show that there exists a uniform $\delta>0$, whenever $d(z, w)<\delta$ :

$$
B_{h^{N}}(z, w)=B_{h^{N}, l o c}^{k}(z, w)+O\left(N^{m-k-1}\right)
$$


This means the difference between the local Bergman kernel and the global one is up to a small error term. Now, in our noncompact case, if we can prove (31) for any fixed compact subset $K$, then Theorem 4.1 follows if we combine (29) and (31) and let $z=w$. In order to prove this, now choose $\chi$ as the cut-off function equal to 1 in a neighborhood of $z$ which is large enough to contain $w$, let's apply (30) to $u=B_{h^{N}}(z, w)$, then we have the reminder $O\left(N^{-k-1}\right)\left\|B_{h^{N}}\right\|$. Furthermore, if we use the well-known estimate $B_{h^{N}} \leq C N^{m}$, we have:

$$
B_{h^{N}}(z, w)=\left\langle\chi B_{h^{N}}, B_{h^{N}, l o c}^{k}\right\rangle_{h^{N}}+O\left(N^{m-k-1}\right)
$$

Next let's estimate

$$
v_{N}(z, w)=\chi B_{h^{N}}^{k}(z, w)-\left\langle\chi B_{h^{N}}^{k}, B_{h^{N}}\right\rangle_{h^{N}}
$$

Since the inner product is the Bergman projection, $v_{N}(z, w)$ will be the $L^{2}$-minimal solution to the $\bar{\partial}$-equation $\bar{\partial} u_{N}=\bar{\partial}\left(\chi B_{h^{N}}^{k}\right)$. Because of the curvature assumption $\operatorname{Ric}(h)>$ $-\gamma \operatorname{Ric}(\omega)$, we can apply the Hömander $L^{2}-\bar{\partial}$ estimate De](Theorem 5.1). Then we have $\left\|u_{N}(z, w)\right\|_{h^{N}}^{2} \leq O\left(k^{-\infty}\right)$. Then by Cauchy integral formula in a ball around $z$ of radius $1 / N^{1 / 2}$, we have the pointwise estimate $\left|u_{N}(z, w)\right|^{2} \leq O\left(k^{-\infty}\right)$. Then, (31) follows this estimate and identity (32). Thus, the Theorem 4.1 follows. Note that, in our modifications, we have to restrict the Bergman kernel to any compact subset to ensure the existence the uniform $\delta$ and we also need the curvature assumption to ensure Hömander $L^{2}-\bar{\partial}$ estimate. More details about the Kähler toric case can be found in SZ.

Now let's turn to the proof of Theorem 1.2 .

Proof. First, recall equation (26), if we use the general formula (24), we have:

$$
\mathcal{N}_{h^{N}} f(x)=\left.\int_{\mathbb{R}^{m}} \hat{f}(\xi) e^{i\left\langle\xi, N^{-1} D_{\theta}\right\rangle} B_{h^{N}}\left(e^{i \theta} z, z\right)\right|_{\theta=0} d \xi=\int_{\mathbb{R}^{m}} \hat{f}(\xi) B_{h^{N}}\left(z, z e^{i N^{-1} \xi}\right)
$$

Next, let's plug in identity (28), we have:

$$
\mathcal{N}_{h^{N}} f(x)=\int_{\mathbb{R}^{m}} \hat{f}(\xi) e^{N\left(F\left(e^{i N^{-1} \xi}|z|^{2}\right)-F\left(|z|^{2}\right)\right)} A_{N}\left(z, z e^{i N^{-1} \xi}\right)
$$

Next, note that:

$$
\begin{aligned}
& F\left(e^{i N^{-1} \xi}|z|^{2}\right)-F\left(|z|^{2}\right)=\int_{0}^{1} \frac{d}{d t} F\left(e^{i t N^{-1}} \xi|z|^{2}\right) d t=i N^{-1} \int_{0}^{1}\left\langle\nabla_{\xi} F\left(e^{i t N^{-1} \xi+\rho}\right), \xi\right\rangle d t \\
& =i N^{-1}\left\langle\nabla_{\rho} F\left(e^{\rho}\right), i \xi\right\rangle-N^{-2} \int_{0}^{1}(t-1)^{2} \nabla_{\rho}^{2} F\left(e^{i t N^{-1} \xi+\rho}\right)(i \xi)^{2} / 2 d t i N^{-1}\langle\mu(z), \xi\rangle= \\
& +\frac{1}{2}(i N)^{2} \nabla_{\rho}^{2}\left(F\left(e^{\rho}\right)\right)(i \xi)^{2}+O\left(N^{-3}\right)=i N^{-1}\langle\mu(z), \xi\rangle-\frac{1}{2} N^{-2}\left\langle H_{z} \xi, \xi\right\rangle+O\left(N^{-3}\right)
\end{aligned}
$$

Hence,

$$
\mathcal{N}_{h^{N}} f(x)=\int_{\mathbb{R}^{m}} \hat{f}(\xi) e^{i\langle\mu(z), \xi\rangle} e^{-\frac{1}{2} N^{-1}\left\langle H_{z} \xi, \xi\right\rangle+O\left(N^{-2}\right)} A_{N}\left(z, z e^{i N^{-1} \xi}\right)
$$

By Taylor expansion, we have:

$$
e^{-\frac{1}{2} N^{-1}\left\langle H_{z} \xi, \xi\right\rangle+O\left(N^{-2}\right)}=1-\frac{1}{2} N^{-1}\left\langle H_{z} \xi, \xi\right\rangle+O\left(N^{-2}\right)
$$


Now combine equations (35) and (36), if we plug in the asymptotics of $A_{N}\left(z, z e^{i N^{-1} \xi}\right)$ in Theorem 4.1 and the fact that $\mu(z)=x$, then by Fourier transformation, we have:

$$
\mathcal{N}_{h^{N}} f(x)=N^{m} f(x)+N^{m-1}\left[-\frac{1}{2}\left\langle H_{z} D_{x}, D_{x}\right\rangle f(x)+a_{1}(z, z) f(x)\right]+O\left(N^{m-2}\right)
$$

where

$$
H_{z}=\nabla_{\rho}^{2}\left(\varphi\left(e^{\rho}\right)\right), \quad z=e^{\frac{\rho}{2}+i \theta}
$$

is the Hessian of the Kähler potential. Since $f(x)$ has compact support $K$, because of the properness of the moment map $\mu, \mu^{-1}(K) \subset M$ will be a compact set, hence, we can divide (37) by the complete asymptotics of $B_{h^{N}}(z, z)$ in Theorem 4.1, then the theorem follows directly.

4.3. Scaling asymptotics. Let's turn to the proof of Theorem 1.3 ,

Proof. Now let's prove the first part of the theorem. Denote $z$ and $\tilde{z}$ such that $\mu(z)=x$ and $\mu(\tilde{z})=x / N$. First, regarding the Hessian matrix $H_{z}$ defined by (38), we claim:

$$
\left(M(x)-N H_{\tilde{z}}\right)+O\left(N^{-2}\right)=\left(M(x)-H_{z}\right) N^{-1}
$$

To prove this identity, first by definitions, we have: $x / N=\mu(\tilde{z})=\nabla_{\rho}\left(\varphi\left(e^{\rho}\right)\right)=\varphi^{\prime}\left(|\tilde{z}|^{2}\right)|\tilde{z}|^{2}$ and $x=\mu(z)=\varphi^{\prime}\left(|z|^{2}\right)|z|^{2}$. For any fixed $x, x / N$ will be contained in a compact subset $K \subset P$ for all $N$, hence $\mu^{-1}(K)$ is a compact subset of $M$ because of the properness of $\mu$, which implies that $\varphi^{\prime}\left(|\tilde{z}|^{2}\right)$ and $\varphi^{\prime}\left(|z|^{2}\right)$ are uniformly bounded, hence,

$$
|\tilde{z}|^{2}=O\left(N^{-1}\right), \quad|z|^{4}=N^{2}|\tilde{z}|^{4}+O\left(N^{-1}\right)
$$

In addition, in view of (38), we have $H_{z}=\varphi^{\prime \prime}\left(e^{\rho}\right) e^{2 \rho}+\varphi^{\prime}\left(e^{\rho}\right) e^{\rho}=\varphi^{\prime \prime}\left(|z|^{2}\right)|z|^{4}+M(\mu(z))$. Since $\mu(\tilde{z})=x / N$ and $\mu(z)=x$, then $M(x)-H_{z}=-\varphi^{\prime \prime}\left(|z|^{2}\right)|z|^{4}$ and $M(x)-N H_{\tilde{z}}=$ $-N \varphi^{\prime \prime}\left(|\tilde{z}|^{2}\right)|\tilde{z}|^{4}$. Thus our claim is equivalent to:

$$
\varphi^{\prime \prime}\left(|z|^{2}\right)|z|^{4}=N^{2} \varphi^{\prime \prime}\left(|\tilde{z}|^{2}\right)|\tilde{z}|^{4}+O\left(N^{-1}\right)
$$

In fact, this is the Taylor expansion of $\varphi^{\prime \prime}\left(|z|^{2}\right)$ at $|\tilde{z}|^{2}: \varphi^{\prime \prime}\left(|z|^{2}\right)|z|^{4}=\varphi^{\prime \prime}\left(|\tilde{z}|^{2}\right)|z|^{4}+O\left(|\tilde{z}|^{2}\right)=$ $\varphi^{\prime \prime}\left(|\tilde{z}|^{2}\right) N^{2}|\tilde{z}|^{4}+O\left(N^{-1}\right)$. In this last equality, we use the fact $|z|^{4}=N^{2}|\tilde{z}|^{4}+O\left(N^{-1}\right)$.

Next, let's denote $f_{N}(x)=D_{N} f(x)$. Then:

$$
\begin{aligned}
& D_{\frac{1}{N}} S_{h^{N}} D_{N} f(x)=D_{\frac{1}{N}} S_{h^{N}} f_{N}(x) \\
& =D_{\frac{1}{N}}\left[\left(\int_{\mathbb{R}^{m}} \widehat{f_{N}}(\xi) e^{N\left(F\left(e^{i N^{-1} \xi}\left|\mu^{-1}(x)\right|^{2}\right)-F\left(\left|\mu^{-1}(x)\right|^{2}\right)\right)} A_{N}\left(\mu^{-1}(x), \mu^{-1}(x) e^{i \frac{\xi}{N}}\right)\right) / B_{h^{N}}\left(\mu^{-1}(x), \mu^{-1}(x)\right] d \xi\right.
\end{aligned}
$$

Use the fact $\widehat{f_{N}}=N^{-m} \hat{f}(\xi / N)$ and change variables to $\eta=\xi / N$ to get:

$$
=D_{\frac{1}{N}}\left[\left(\int_{\mathbb{R}^{m}} \widehat{f}(\eta) e^{N\left(F\left(e^{i \eta}\left|\mu^{-1}(x)\right|^{2}\right)-F\left(\left|\mu^{-1}(x)\right|^{2}\right)\right)} A_{N}\left(\mu^{-1}(x), \mu^{-1}(x) e^{i \eta}\right)\right) / B_{h^{N}}\left(\mu^{-1}(x), \mu^{-1}(x)\right] d \eta\right.
$$

Now do the final dilation to get:

$$
=\left[\left(\int_{\mathbb{R}^{m}} \widehat{f}(\eta) e^{N\left(F\left(e^{i \eta}\left|\mu^{-1}\left(\frac{x}{N}\right)\right|^{2}\right)-F\left(\left|\mu^{-1}\left(\frac{x}{N}\right)\right|^{2}\right)\right)} A_{N}\left(\mu^{-1}\left(\frac{x}{N}\right), \mu^{-1}\left(\frac{x}{N}\right) e^{i \eta}\right)\right) / B_{h^{N}}\left(\mu^{-1}\left(\frac{x}{N}\right), \mu^{-1}\left(\frac{x}{N}\right)\right] d \eta\right.
$$


By Taylor expansion, we have: $\frac{x}{N} \cdot e^{i \eta}=\frac{x}{N}+\frac{x}{N} \cdot i \eta-\left\langle M\left(\frac{x}{N}\right) \eta, \eta\right\rangle / 2+O\left(N^{-2}\right)$, then we can rewrite (34) as:

$$
F\left(e^{i \eta}\left|\mu^{-1}\left(\frac{x}{N}\right)\right|^{2}\right)-F\left(\left|\mu^{-1}\left(\frac{x}{N}\right)\right|^{2}\right)=\left(e^{i \eta}-1\right) \cdot \frac{x}{N}+\left\langle\left[M\left(\frac{x}{N}\right)-H_{\tilde{z}}\right] \eta, \eta\right\rangle / 2+O\left(N^{-3}\right)
$$

Combine (40) and (41), we have:

$$
\left(D_{\frac{1}{N}} S_{h^{N}} D_{\frac{1}{N}}^{-1}\right) f(x)=\int_{\mathbb{R}^{m}} \widehat{f}(\eta) e^{\left(e^{i \eta}-1\right) \cdot x} \tilde{A}_{N}\left(\tilde{z}, \tilde{z} e^{i \eta}\right) / B_{h^{N}}(\tilde{z}, \tilde{z}) d \eta
$$

where $\tilde{A}_{N}=A_{N} \cdot e^{\left\langle\left(M(x)-N H_{\tilde{z}}\right) \eta, \eta\right\rangle / 2+O\left(N^{-2}\right)}$. If we plug in claim (39) and asymptotics of $A_{N}$, we have: $\tilde{A}_{N}=N^{m}\left(1+a_{1}\left(\tilde{z}, \tilde{z} e^{i \eta}\right) N^{-1}+\cdots\right) \cdot e^{\left\langle\left(M(x)-H_{z}\right) \eta, \eta\right\rangle / 2 N+O\left(N^{-2}\right)}$. If we use the Taylor expansion of $a_{1}\left(\tilde{z}, \tilde{z} e^{i \eta}\right)$ and $e^{\left\langle\left(M(x)-H_{z}\right) \eta, \eta\right\rangle / 2 N+O\left(N^{-2}\right)}$ at 0 simultaneously, and plug in the asymptotics of $B_{h^{N}}$, then $\tilde{A}_{N}\left(\tilde{z}, \tilde{z} e^{i \eta}\right) / B_{h^{N}}(\tilde{z}, \tilde{z})=1+\left[\left\langle\left(M(x)-H_{z}\right) \eta, \eta\right\rangle / 2+a_{1}(0)\right] N^{-1}+$ $O\left(N^{-2}\right)$. Hence, combine this identity and equation (42), the first term of the complete asymptotic expansion of $\left(D_{\frac{1}{N}} S_{h^{N}} D_{\frac{1}{N}}^{-1}\right) f(x)$ is given by:

$$
\int_{\mathbb{R}^{m}} \widehat{f}(\eta) e^{\left(e^{i \eta}-1\right) \cdot x} d \eta=\sum_{|\alpha|=0}^{\infty} e^{-x} \frac{x^{\alpha}}{\alpha !} \int_{\mathbb{R}^{m}} \widehat{f}(\eta) e^{i \alpha \eta} d \eta=e^{-x} \sum_{|\alpha|=0}^{\infty} f(\alpha) \frac{x^{\alpha}}{\alpha !}=S_{h_{B F}^{1}} f(x)
$$

And the second term which is the coefficient of $N^{-1}$ is given by:

$b_{1}(x)=\int_{\mathbb{R}^{m}} \widehat{f}(\eta) e^{\left(e^{i \eta}-1\right)}\left[\left\langle\left(M(x)-H_{z}\right) \eta, \eta\right\rangle / 2+a_{1}(0)\right] d \eta=\left[\frac{1}{2}\left\langle\left(M(x)-H_{z}\right) D_{x}, D_{x}\right\rangle+a_{1}(0)\right] S_{h_{B F}^{1}}(f)(x)$

which completes the proof.

For the second part, we have to use the notation of Slice coordinates [SoZ]. Assume $\left\{F_{j}\right\}_{j=1}^{m}$ are all facets which have a common vertex at 0 . Let $F=\cap_{j=1}^{k} F_{j}$ be a $(m-k)$-dimensional (open) face. Then by angle-action coordinates, we can split the torus action into two parts $\mathbb{T}^{m}=\mathbb{T}^{k} \times \mathbb{T}^{m-k}$, where $\mathbb{T}^{k}$ is the stabilizer at $\mu^{-1}(F)$. And we can split all coordinates into two parts corresponding to $\mathbb{T}^{k}$ and $\mathbb{T}^{m-k}$ action. First, we can split $z=\left(z^{\prime}, z^{\prime \prime}\right) \in \mathbb{C}^{k} \times \mathbb{C}^{m-k}$ where $\left(0, z^{\prime \prime}\right)$ is a local coordinate of the submanifold $\mu^{-1}(\bar{F})$, where $\bar{F}$ is the closure of $F$. In addition, we have $\mu(z)=\left(\mu^{\prime}(z), \mu^{\prime \prime}(z)\right)$, and $\mu^{\prime \prime}(z)$ is the moment map for the Hamiltonian $\mathbb{T}^{m-k}$ action on the Kähler toric submanifold with $z^{\prime}=0$.

First, using Taylor expansion, we have:

$$
F\left(|z|^{2}\right)=F\left(\left|z^{\prime}\right|^{2},\left|z^{\prime \prime}\right|^{2}\right)=F\left(0,\left|z^{\prime \prime}\right|^{2}\right)+F^{\prime}\left(0,\left|z^{\prime \prime}\right|^{2}\right)\left|z^{\prime}\right|^{2}+O\left(\left|z^{\prime}\right|^{4}\right)
$$

where $F^{\prime}$ is the $z^{\prime}$-derivative of $F$. If denote $\mu(z)=\left(x^{\prime} / N, x^{\prime \prime}\right)$, then it's easy to see that $\left|z^{\prime}\right|^{2}=O\left(N^{-1}\right)$. Now denote $\varsigma=(\eta, \xi / N)$, then by the similar argument as the one to get (40), we have:

$$
\left(\hat{D}_{\frac{1}{N}} S_{h^{N}} \hat{D}_{\frac{1}{N}}^{-1}\right) f\left(x^{\prime}, x^{\prime \prime}\right)=\int_{\mathbb{R}^{m}} \widehat{f}(\xi, \eta) e^{N\left(F\left(e^{i \varsigma}|z|^{2}\right)-F\left(|z|^{2}\right)\right)} A_{N}\left(z, z e^{i \varsigma}\right) / B_{h^{N}}\left(z, z e^{i \varsigma}\right) d \xi d \eta
$$

First, use the fact that $\mu^{\prime \prime}(z)$ is the moment map for the Hamiltonian $\mathbb{T}^{m-k}$ action on the Kähler toric submanifold with $z^{\prime}=0$, we have,

$$
F\left(0,\left|z^{\prime \prime}\right|^{2}\right)-F\left(0, e^{i N^{-1} \xi}\left|z^{\prime \prime}\right|^{2}\right)=i N^{-1}\left\langle\mu^{\prime \prime}(z), \xi\right\rangle+O\left(N^{-2}\right)
$$


Second, use the Taylor expansion,

$$
F^{\prime}\left(0, e^{i N^{-1} \xi}\left|z^{\prime \prime}\right|^{2}\right) e^{i \eta}\left|z^{\prime}\right|^{2}-F^{\prime}\left(0,\left|z^{\prime \prime}\right|^{2}\right)\left|z^{\prime}\right|^{2}=\mu^{\prime}(z)\left(e^{i \eta}-1\right)+O\left(N^{-2}\right)
$$

Plug these two equations to (43) and plug in the asymptotics of $A_{N} / B_{h^{N}}$, we have:

$$
\begin{aligned}
& \left(\hat{D}_{\frac{1}{N}} S_{h^{N}} \hat{D}_{\frac{1}{N}}^{-1}\right) f\left(x^{\prime}, x^{\prime \prime}\right)=\int_{\mathbb{R}^{m}} \hat{f}(\xi, \eta) e^{i\left\langle x^{\prime \prime}, \xi\right\rangle} e^{x^{\prime}\left(e^{i \eta}-1\right)} d \xi d \eta+O\left(N^{-1}\right) \\
& =e^{-x^{\prime}} \sum_{|\alpha|=0}^{\infty} \frac{x^{\prime \alpha}}{\alpha !} f\left(\frac{\alpha}{N}, x^{\prime \prime}\right)+O\left(N^{-1}\right)=S_{h_{B F}^{1}}\left(f_{x^{\prime \prime}}\right)\left(x^{\prime}\right)+O\left(N^{-1}\right)
\end{aligned}
$$

Follow this step, if we expand more terms in $\left(\hat{D}_{\frac{1}{N}} S_{h^{N}} \hat{D}_{\frac{1}{N}}^{-1}\right) f\left(x^{\prime}, x^{\prime \prime}\right)$, we will get the complete asymptotics.

\section{Examples of GENERALIZED SZASz ANALYTiC FUnCTIONS}

5.1. $B^{m}$ with the Bergman metric. In this subsection, we will compute the generalized Szasz analytic functions for $B^{m}$ with the Bergman metric. We will see that this generalized function is related to the negative Binomial or Pascal distribution.

In this case, the Kähler potential is given by:

$$
\varphi(z)=-\log \left(1-|z|^{2}\right)=\varphi(\rho)=-\log \left(1-e^{\rho}\right)
$$

where $|z|^{2}=\left|z_{1}\right|^{2}+\cdots+\left|z_{m}\right|^{2}<1$.

So the moment map is:

$$
\mu\left(z_{1}, \ldots, z_{m}\right)=\nabla_{\rho} \varphi(\rho)=\left(\frac{\left|z_{1}\right|^{2}}{1-|z|^{2}}, \cdots, \frac{\left|z_{m}\right|^{2}}{1-|z|^{2}}\right)
$$

Furthermore, denote $\|x\|=\sum_{j=1}^{m} x_{j}$ and $x_{j}>0$, then we have the sympletic potential:

$$
u(x)=\sum_{j=1}^{m} x_{j} \log x_{j}-(1+\|x\|) \log (1+\|x\|)
$$

First, we have the Bergman kernel for the Fubini-Study metric [Z1]:

$$
B_{h_{F S}^{N}}(z, w)=\frac{(N+m) !}{N !}\left(\frac{(1+z \bar{w})}{\sqrt{1+|z|^{2}} \sqrt{1+|w|^{2}}}\right)^{N}
$$

Since $B^{m}$ is dual to $\mathbb{C P}^{m}$ as a symmetric space in the sense of S. Helgason [H], its Bergman kernel should replace the term $1+|z|^{2}$ in $B_{h_{F S}^{N}}$ by $1-|z|^{2}$. Furthermore, the hyperplane line bundle $\mathcal{O}(1) \rightarrow \mathbb{C P}^{m}$ carries a natural Fubini-Study metric which makes it to be positive. Hence the canonical bundle of $\mathbb{C P}^{m}$ which is isomorphic to $\mathcal{O}(-m-1)$ will be negative and the tangent bundle will be positive when they are equipped with the metric induced by Fubini-Study metric. By the duality again, the reverse is true on $B^{m}$. Hence, we also need to change the sign of the exponent:

$$
B_{h^{N}}(z, w)=\frac{(N+m) !}{N !}\left(\frac{(1-z \bar{w})}{\sqrt{1-|z|^{2}} \sqrt{1-|w|^{2}}}\right)^{-N}
$$


On the diagonal we have

$$
B_{h^{N}}(z, z)=\frac{(N+m) !}{N !}
$$

Now let's compute the generalized Szasz analytic functions for the unit disc, the same conclusion should be valid for general cases. In the unit disc case, the orthonormal basis of is:

$$
\left\{e_{j}=\sqrt{\frac{\Gamma(j+N)}{j ! \Gamma(N-1)}} z^{j}, \quad j \geq 0, \quad N \geq 2\right\}
$$

Hence, if we plug (45) into to the definition of $S_{h^{N}}(f)(x)$, we have:

$$
S_{h^{N}}(f)(x)=\frac{1}{B_{h^{N}}(z, z)} \sum_{j=0}^{\infty} f\left(\frac{j}{N}\right) \frac{e^{N\left(u(x)+\left\langle\frac{j}{N}-x, \nabla u(x)\right\rangle\right)}}{\left\|z^{j}\right\|_{h^{N}}^{2}}=\frac{(1+x)^{-N}}{B_{h^{N}}(z, z)} \sum_{j=0}^{\infty} f\left(\frac{j}{N}\right) \frac{\left(\frac{x}{1+x}\right)^{j}}{\left\|z^{j}\right\|_{h^{N}}^{2}}
$$

Now plug in $B_{h^{N}}(z, z)$ (46) and $\left\|z^{j}\right\|_{h^{N}}^{2}$ (47) to get:

$$
S_{h^{N}}(f)(x)=\frac{N-1}{N+1} \cdot(1+x)^{-N} \sum_{j=0}^{\infty}(N)_{j} f\left(\frac{j}{N}\right) \frac{\left(\frac{x}{1+x}\right)^{j}}{j !}
$$

where $(N)_{j}=N(N+1) \cdots(N+j-1)$. Hence, in view of Theorem 1.2, we have the following estimate :

$$
(1+x)^{-N} \sum_{j=0}^{\infty}(N)_{j} f\left(\frac{j}{N}\right) \frac{\left(\frac{x}{1+x}\right)^{j}}{j !} \rightarrow f(x), \text { as } N \rightarrow \infty
$$

Next, we will see that our generalized Szasz analytic expansion relates the Bergman metric of $B_{m}$ with the negative binomial or Pascal distribution in the same sense that the Bernstein polynomial is related to the binomial distribution. Let's recall that the Pascal distribution $X$ is the probability distribution of failures before the first success in a series of independent and identically distributed Bernoulli trials. Denote $q=1-p$, then for any integer $j \geq N$, $P(X=j)$ is the probability of the event that there are exact $N-1$ successful trials among $X_{1}, X_{2}, \ldots, X_{j-1}$ and $X_{j}$ is successful trial. Hence

$$
P(X=j)=\left(\begin{array}{c}
j-1 \\
N-1
\end{array}\right) p^{N} q^{j-N}, j=N, N+1, N+2 \ldots
$$

THEOREM 5.1. If $f(x)$ is continuous with compact support on $[0, \infty)$, then we have:

$$
S_{h^{N}}(f)(x)=E\left(f\left(\frac{X}{N}\right)\right)+O\left(\frac{1}{N}\right)
$$

where $X$ is Pascal distribution and the probability of the successful trial is $p=\frac{1}{1+x}$.

Proof. Note that if the random variable $\xi$ has the distribution $F(x)$ and $g(x)$ is any Borel measurable function, then we have the identity: $E g(\xi)=\int_{-\infty}^{\infty} g(x) d F(x)$. Hence,

$$
\begin{gathered}
E\left(f\left(\frac{X}{N}\right)\right)=\sum_{j \geq N} f\left(\frac{j}{N}\right)\left(\begin{array}{c}
j-1 \\
N-1
\end{array}\right) p^{N} q^{j-N}=p^{N} \sum_{j=0}^{\infty} f\left(\frac{j}{N}\right)\left(\begin{array}{c}
N+j-1 \\
N-1
\end{array}\right) q^{j} \\
=(1+x)^{-N} \sum_{j}(N)_{j} f\left(\frac{j}{N}\right) \frac{\left(\frac{x}{1+x}\right)^{j}}{j !}
\end{gathered}
$$


In the last step, we plug in $p=\frac{1}{1+x}$ and $q=\frac{x}{1+x}$. Hence, the theorem follows if we combine this with equation (48).

5.2. Kepler manifolds with incomplete metrics. In this subsection, we will see that Theorem 1.2 is also valid for some incomplete toric Kähler manifolds (maybe have singularities), the examples are the complex 1,2 and 3-dimensional Kepler manifolds GL, Ra]. The Kepler manifold is a complex $m$-dimensional symplectic manifold $(M, \omega)$, where $M=$ $T^{*} S^{m} \backslash 0$ is the cotangent bundle to the real $m$-dimensional sphere minus its zero section endowed with the standard symplectic form $\omega$. This may further be identified with:

$$
M=\left\{(e, t) \in \mathbb{R}^{m+1} \times \mathbb{R}^{m+1} \mid\langle e, e\rangle=1,\langle t, e\rangle=0, t \neq 0\right\}
$$

where the inner product is the standard scalar product on $\mathbb{R}^{m+1}$. If we introduce $z=$ $|t| e+i t \in \mathbb{C}^{m+1}$, then the Kepler manifold is identified to the isotropic cone:

$$
\mathcal{C}_{m}=\left\{z \in \mathbb{C}^{m+1} \mid z \cdot z=z_{1}^{2}+\cdots+z_{m+1}^{2}=0, z \neq 0\right\} \subset \mathbb{C}^{m+1}
$$

In addition, the symplectic form $\omega$ is indeed an incomplete Kähler form:

$$
\omega=\frac{i}{2} \partial \bar{\partial}|t|
$$

with Kähler potential $\varphi=|t|=\sqrt{z \bar{z} / 2}=\frac{\sqrt{2}}{2} e^{\rho / 2}$. Denote $L$ as the holomorphic line bundle over $M$ such that the first Chern class $c_{1}(L)=\omega$, then the Bergman kernel is given by:

$$
B_{h^{N}}(z, z)=2^{m-1} N^{m} e^{-N|t|} \sum_{j=0}^{\infty} \frac{(j+m-2) !}{(2 j+m-2) !} \frac{(N|t|)^{2 j}}{j !}
$$

where $\left\{z^{j}\right\}_{j=0}^{\infty}$ is a basis of $H_{L^{2}}^{0}\left(M, L^{N}\right)$. In GL, the authors compute the complete asymptotic of Bergman kernel for the Kepler manifolds:

$$
B_{h^{N}}(z, z)=N^{m}+\frac{(m-2)(m-1)}{2|t|} N^{m-1}+\sum_{k=2}^{m-2} \frac{2 a_{k}}{|t|^{k}} N^{m-k}+R_{N}(|t|)
$$

where $a_{k}, k \geq 2$ can be computed explicitely by recursive formulas and $R_{N}(|t|)=O\left(e^{-c N}\right)$ as $m \rightarrow \infty$ uniformly with respect to $|t| \geq \delta>0$.

When $m=1$, there is a natural $\mathbb{T}^{1}$ action on $\mathcal{C}_{1}$ which maps $z$ to $e^{-i \theta} z$, i.e., in the real coordinate, $(e, t)$ is mapped to $(\cos \theta e+\sin \theta t, \cos \theta t-\sin \theta|t| e)$ and the Kähler potential $\varphi=|t|^{2}$ is invariant under this action. Hence, $\mathcal{C}_{1}$ is a noncompact incomplete toric Kähler manifold with a singularity. Note that all formula such as the moment map, symplectic potential are still valid for singular toric varieties [BGL]. Hence, the moment map is given by

$$
x=\nabla_{\rho} \varphi=|t| / 2
$$

Now, if we plug equations (50) and (52) into the definition of the generalized Szasz analytic functions, for the smooth function $f(x)$ with compact support on $[\delta,+\infty)$, we have

$$
S_{h^{N}, \mathcal{C}_{1}}(f)(x)=\frac{2 N e^{-2 N x}}{N^{1}+R_{N}(|x|)} \sum_{j=0}^{\infty} f\left(\frac{j}{N}\right) \frac{(2 N x)^{2 j}}{(2 j) !}
$$


Now let's apply the complete asymptotics of Bergman kernel (51), it's easy to see that:

$$
S_{h^{N}, \mathcal{C}_{1}}(f)(x)=2 e^{-2 N x} \sum_{j=0}^{\infty} f\left(\frac{j}{N}\right) \frac{(2 N x)^{2 j}}{(2 j) !} \rightarrow f(x)
$$

as $N \rightarrow \infty$. In the last estimate, we use the fact that the classical Szasz analytic function (1) converges to $f(x)$ as $N \rightarrow \infty$.

When $m=2$, by changing variables, $\mathcal{C}_{2}$ can be rewritten as $\left\{\left(z_{1}, z_{2}, z_{3}\right) \in \mathbb{C}^{3}-\{0\} \mid z_{1}^{2}=\right.$ $\left.z_{2} z_{3}\right\}$, this cone is the classical example in p.5 in $[\mathrm{F}]$ and have quotient singularity at $(0,0)$. The moment polytope is given by:

$$
\left\{(x, y) \in \mathbb{R}^{2} \mid x \geq 0,0 \leq y \leq 2 x,(x, y) \neq(0,0)\right\}
$$

By the same computation as in $\mathcal{C}_{1}$, it's easy to see that:

$$
S_{h^{N}, \mathcal{C}_{2}}(f)(x)=\frac{2 N^{2} e^{-2 N x}}{N^{2}+R_{N}(|x|)} \sum_{\alpha \in \mathbb{Z}_{+}^{2}} f\left(\frac{\alpha}{N}\right) \frac{(2 N x)^{2 \alpha}}{(2 \alpha) !}
$$

where $f(x) \in C_{c}^{\infty}\left([\delta,+\infty)^{2}\right), x=\left(x_{1}, x_{2}\right)$ and $\alpha=\left(\alpha_{1}, \alpha_{2}\right)$ is multi-index. Hence, we have the similar asymptotic as the one in $\mathcal{C}_{1}$.

When $m=3, \mathcal{C}_{3}$ can be rewritten as $\left\{\left(z_{1}, z_{2}, z_{3}, z_{4}\right) \in \mathbb{C}^{4}-\{0\} \mid z_{1} z_{2}=z_{3} z_{4}\right\}$ which is the complex 3-dimensional Conifold discussed in subsection 2.1.2, Hence,

$$
S_{h^{N}, \mathcal{C}_{3}}(f)(x)=\frac{2 N^{3} e^{-2 N x}}{N^{3}+\frac{1}{2 N x}+R_{N}(|x|)} \sum_{\alpha \in \mathbb{Z}_{+}^{3}} \frac{2 j+2}{2 j+1} f\left(\frac{\alpha}{N}\right) \frac{(2 N x)^{2 \alpha}}{(2 \alpha) !}
$$

where $f(x) \in C_{c}^{\infty}\left([\delta,+\infty)^{3}\right)$. By the similar argument above, we also have $S_{h^{N}, \mathcal{C}_{3}}(f)(x) \rightarrow$ $f(x)$ as $N \rightarrow \infty$.

Acknowledgements: I am sincerely grateful to Prof. S. Zelditch for his patience to guide me to this beautiful math world. This paper will never come out without his help and many suggestions.

\section{REFERENCES}

[A1] M. Abreu, Kähler geometry of toric varieties and extremal metrics, Internat. J. Math. 9 (1998), no. $6,641-651$.

[A2] M. Abreu, Kähler geometry of toric manifolds in symplectic coordinates, Symplectic and contact topology: interactions and perspectives (Toronto, ON/Montreal, QC, 2001), Fields Inst. Commun., 35, Amer. Math. Soc., Providence, RI, 2003, pp. 1-24.

[Be] S. Bernstein, Démonstration du théorème de Weierstrass, fondée sur le calcul des probabilités, Comm. Soc. Math. Kharkov = Charkow Ges. (2) 13 (1912), 1-2. JFM 43.0301 .03

[Bo] V. Bouchard, Lectures on complex geometry, Calabi-Yau manifolds and toric geometry, arXiv:0702063.

[BBS] R. Berman, B. Berndtsson and J. Sjöstrand, A direct approach to Bergman Kernel asymptotics for positive line bundles, Ark. Mat., 46 (2008), 197-217.

[BGL] D. Burns, V. Guillemin, E. Lerman, Kähler metrics on singular toric varieties, Pacific Journal of Mathematics. Vol. 238 (2008), No. 1, 27-40

[CdS] A. Cannas da Silva, Lectures on Symplectic Geometry, volume 1764 of Lecture Notes in Mathematics-Springer-Verlag, Berlin.

[CO] P. Candelas, X. C. de la Ossa, Comments on conifolds, Nuclear Physics B, Volume 342, Issue 1, p. 246-268. 
[CY] S.Y. Cheng and S.T. Yau, On the existence of a complete Kähler metric on noncompact complex manifolds and the regularity of Feffermans equation, Comm. Pure Appl. Math. 33 (1980), 507C544.

[De] J.P. Demailly, $L^{2}$ Vanishing Theorems for Positive Line Bundles and Adjunction Theory, arXiv:9410022v3 .

[Del] T. Delzant, Hamiltoniens periodic et images convexes de lapplication moment, Bul. Soc. math. France 116 (1988), 315-339.

[DO] K. Diederich and T. Ohsawa, An estimate for the Bergman distance on pseudoconvex domains, Ann. Math. 141 (1995), 181C190.

[F] W. Fulton, Introduction to Toric Varieties, Princeton University Press, Princeton, NJ, 1993.

[G] V. Guillemin, Moment Maps and Combinatorial Invariants of Hamiltonian $\mathbb{T}^{n}$-spaces, Process in Mathematics 122, Birkhäuser, 1994

[GL] T. Gramchev and A. Loi, TYZ expansion for the Kepler manifold, Comm. Math. Phys., to appear.

$[\mathrm{H}] \quad$ S. Helgason, Differential geometry and symmetric spaces, Pure and Appl. Math., vol. 12, Academic Press, New York, 1962.

[INOV] A. Iqbal, N. Nekrasov, A. Okounkov and C. Vafa, Quantum Foam and Topological Strings, Journal of High Energy Physics, Issue 04, pp. 011 (2008).

[Le] E. Lerman, Symplectic cuts, Mathematical Research Letters 2, 247C258 (1995).

[LS] E. Lerman and S. Tolman, Hamiltonian torus actions on symplectic orbifolds and toric varieties. Trans. Amer. Math. Soc. 349(10): 4201-4230, 1997.

[Lu] Z. Lu, On the lower order terms of the asymptotic expansion of Tian-Yau-Zelditch. Amer. J. Math. 122 (2000), no. 2, 235C273.

[MS] D. Martelli and J. Sparks, Toric Geometry, Sasaki-Einstein Manifolds and a New Infinite Class of AdS/CFT Duals. Commun. Math. Phys. 262, 51-89(2006).

[P] E. Prato, Convexity properties of the moment map for certain non-compact manifolds, Comm. Anal. Geom. 2 (1994), 267-278.

[Ra] J. H. Rawnsley, Coherent states and Kähler manifolds, The Quarterly Journal of Mathematics (1977), 403-415.

[Re] S. I. Resnick, Adventures in Stochastic Processes, Birkhäuser (Springer), 1992.

[S] O. Szasz, Generalization of S. Bernstein's polynomials to the infinite interval, Journal of Research of the National Bureau of Standards, 45 (1950), 239-245. 99 - 130.

[SZ] B. Shiffman and S. Zelditch, Almost holomorphic sections of ample line bundles over symplectic manifolds, J. Reine Angew. Math. 544 (2002), 181-222.

[SoZ] J. Song and S. Zelditch, Bergman metrics and geodesics in the space of Kähler metrics on toric varieties, arXiv:0707.3082,

[T] G. Tian, On a set of polarized Kahler metrics on algebraic manifolds, J. Differential Geom. 32 (1990), 99-130.

[Z1] S. Zelditch, Bernstein polynomials, Bergman kernels and toric Kähler varieties, J. Symplectic Geom. Vol. 7 (2009), 1-26.

[Z2] S. Zelditch, Szegö kernels and a theorem of Tian, IMRN 6 (1998), 317C331.

Department of Mathematics, Johns Hopkins University, USA

E-mail address: rfeng@math.jhu.edu 